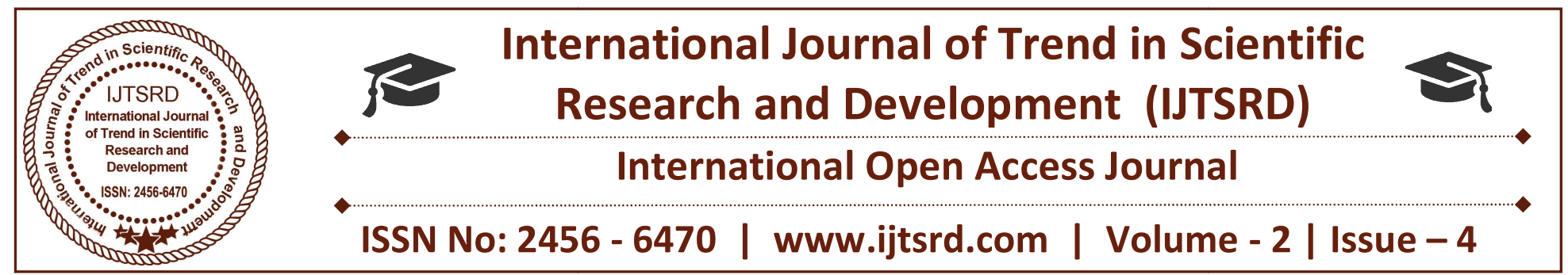

\title{
Implementation of Brain Tumor Extraction Application from MRI Image
}

\author{
Satish Chandra. B \\ Research Scholar, Vinayaka \\ Mission Research Foundation \\ University, Salem, India
}

\author{
Smt K. Satyavathi \\ Research Scholar, Brilliant \\ Grammar School Educational \\ Society's group of Institutions - \\ Integrated Campus, Hyderabad
}

Dr. Krishnanaik Vankdoth
Professor, ECE Dept, Brilliant
Grammar School Educational
Society's group of Institutions-
Integrated Campus, Hyderabad

\begin{abstract}
Medical image process is that the most difficult and rising field currently now a day. Process of MRI pictures is one amongst the part of this field. This paper describes the projected strategy to find \& extraction of tumour from patient's MRI scan pictures of the brain. This technique incorporates with some noise removal functions, segmentation and morphological operations that area unit the fundamental ideas of image process. Detection and extraction of tumor from MRI scan pictures of the brain is finished by victimization MATLAB software package
\end{abstract}

Keywords: Digital Image, MRI, Matlab, Detection, Segmentation, Extraction, Scan

\section{INTRODUCTION}

Large amount of image data is produced in the field of medical imaging in the form of Computed Tomography (CT), Magnetic Resonance Imaging (MRI), and Ultrasound Images, which can be stored in picture archiving and communication system (PACS) or hospital information system. A medium scale hospital with above facilities produces on an average 5 GB to 15 GB of data. So, it is really difficult for hospitals to manage the storing facilities for the same. Moreover, such high data demand for high end network especially for transmitting the images over the network such as in telemedicine. This is significant for telemedicine scenario due to limitations of transmission medium in Information and Communication Technology (ICT) especially for rural area. Image compression is useful in, reducing the storage and transmission bandwidth requirements of medical images. For e.g., an 8-bit grey scale image with $512 \times 512$ pixels requires more than $0.2 \mathrm{MB}$ of storage. If the image is compressed by $8: 1$ compression without any perceptual distortion, the capacity of storage increases 8 times. Compression methods are classified into lossless and lossy methods. In the medical imaging scenario, lossy compression schemes are not generally used. This is due to possible loss of useful clinical information which may influence diagnosis. In addition to these reasons, there can be legal issues. Storage of medical images is generally problematic because of the requirement to preserve the best possible image quality which is usually interpreted as a need for lossless compression.3D MRI contains multiple slices representing all information required about a body part. Some of the most desirable properties of any compression method for 3D medical images include: (i) high lossless compression ratios, (ii) resolution scalability, which refers to the ability to decode the compressed image data at various resolutions, and (iii) quality scalability, which refers to the ability to decode the compressed image at various qualities or signal-to-noise ratios (SNR) up to lossless reconstruction. DICOM is the most comprehensive and accepted version of an imaging communications standard. DICOM format has a header which contains information about the image, imaging modality and information about the patient. The header also contains information about the type of media (CT, MRI, audio recording, etc.) and the image dimensions. Body of DICOM standard contains information 
objects such as medical reports, audio recordings and images. The coding-decoding algorithm must take care of other information in the DICOM file. Also, the algorithms should accept the input image in DICOM format at encoder end and produce DICOM file at decoder end. The size of a graphics file can be minimized in bytes without degrading the quality of the image to an unacceptable level using Image compression. So that more images can be stored in the given memory space. This also minimizes the image and receiving time of the images, say for an example: through Internet. Several methods are there for compressing the images. This is because the statistical properties of the images can be exploited well only by the encoders specially designed for them. Sometimes, some of the inner details in the image can be sacriced for the sake of little more bandwidth or storage space. In other words, lossy compression can be used in such areas. Generally, a text file can be compressed without the introduction of errors up to a certain extent. This is called lossless compression. But after that extent errors are unavoidable. In text and program files it is so important that we use lossless compression because a single error in text or program file will change the meaning of the text or cause the program not to run. A small loss in image compression is always not noticeable. There is no concern till the critical point. Beyond that it's not possible! The compression factor can be high if there is loss tolerance or else it must be less. So, graphic images can be with high compression ratio than that of the text files or program files. Efficient compression is one of the major aspects of image storage. For example an image of 1024 x 1024 x 24 would require the storage memory of $3 \mathrm{MB}$ and needs 7 minutes for transmitting and utilizing in a high speed ISDN (64 Kbit/s). But if the image is compressed at the ratio of 10:1 the memory required for storage would be just $300 \mathrm{~KB}$ and the transmission time drops under 6 seconds. In the time required for sending an uncompressed file through Apple talk network, we can transfer compressed seven $1 \mathrm{MB}$ files to a floppy. In any kind of environment, the large files are always a biggest setback in systems. This shows how desperately we need compression for managing transmittable dimensions. Apart from compression methods, we can also increase the bandwidth but this will not provide efficient outputs. The qualitative transition from simple text to full motion video data and the disk space, transmission bandwidth, and transmission time needed to store and transmit such uncompressed data.

\subsection{MOTIVATION AND PERSPECTIVE}

Digital image processing deals with manipulation of digital images through a digital computer. It is a subfield of signals and systems but focus particularly on images. DIP focuses on developing a computer system that is able to perform processing on an image. The input of that system is a digital image and the system process that image using efficient algorithms, and gives an image as an output. The most common example is Adobe Photoshop. It is one of the widely used applications for processing digital images.

\section{LITERATURE SURVEY}

Bhagwat et al [1] presented a paper that shows comparison of K-means, Fuzzy C-means and Hierarchical clustering algorithms for detection of brain tumor, These Three clustering algorithms Kmeans, fuzzy c-means and hierarchical clustering were tested with MRI brain image in non medical format (.jpg, .png, .bmp etc) as well as DICOM image. It is prove that DICOM images produce more efficient result compare to non medical images. Time required for hierarchical clustering is least and fuzzy c-means is maximum to detect the brain tumor where as K-means algorithm produce more accurate result compared to Fuzzy c-means and hierarchical clustering. Detection of brain tumor involves various stages such as image preprocessing, feature extraction, segmentation and classification. Pulse couple neural network (PCNN) uses for image smoothing, feature extraction and image segmentation and Back Propagation Neural Network (BPNN) is used for classification that classify the image whether it is normal or abnormal.

Roy et al introduce the symmetric analysis to detect the brain tumor by making the calculations on the area of tumor. It $\mathrm{s}$ application with several data sets with different tumor size, intensity and location shows that it can automatically detect and segment the brain tumor. MR images gives better result compare to other technique used in the field of medical science like CT images and X-rays Segmentation is one of the essential tasks in medical area but is boringand time consuming.

Image pre-processing including converting RGB image into grey scale then passing that image to the high pass filter in order to remove noise is done and finally the last we get enhanced image for postprocessing that will include watershed segmentation 
and thresholding as well as morphological operation . For the extraction of text region. Morphological operator is used since text regions are composed of vertical edges, horizontal edges and diagonal edge. At different orientation these text are connected together differently. In recent years the concepts of ontology has taken a wide leap from formal specification to the area of artificial intelligence in the domain of experts system. Ontology has been common on World Wide Web great results in the images having nonuniform contrast distributions. Kharrat et al. proposed an algorithm for detection of brain tumor from MRI images [5]. In this paper, the morphological operations, wavelet decomposition and k-means algorithm for segmentation is implemented to extract tumor area. The results show that the algorithm is feasible and performs very well on MRI images. S. Roy et al. explored a technique to distinguish tumor in brain MRI [6]. In this paper, image enhancement, morphological operations and watershed segmentation are applied. Results demonstrate that Watershed Segmentation can effectively extract a tumor if the parameters are set properly before segmentation. Malakooti et al. proposed a tumor segmentation technique which combines both fuzzy logic and neural networks and extracts the boundary taking into account level set method [7]. The proposed technique gives better results as compared to other existing techniques. M. K. Behera et al. proposed a novel fast and robust fuzzy c-means clustering framework for image segmentation based on local spatial and gray information [8]. This method has low computational time, less complexity and the algorithm is effective and efficient. Cai et al. proposed a fuzzy clustering algorithm that utilizes dependable This concept basically deals with classes, sub-classes and their association from the basic categorization of product along with their features .[4] Aboul Ella Hassanien et al [5] presented review paper that shows how the rough set approach and near set approach are useful to solve various problems in medical imaging such as medical image segmentation, object extraction and image classification. This paper also shows how the rough set framework hybridized with various computing technologies such neural network (NN), support vector machine (SVM) and fuzzy sets . A combination of various computational intelligence technologies in medical image problem has become one of the most promising avenues in image processing research.

Magdi et al [6] used an intelligent Model for brain tumor diagnosis from MRI images .which consist of three different stages such as preprocessing, Feature extraction and classification. Preprocessing used to reduce the noise by filtration and to enhance the MRI image through adjustment and edge detection. Texture features are extracted and principal component analysis (PCA) is applied to reduce the features of the image and finally back propagation neural network (BPNN) based Person correlation coefficient was used to classify the brain image.N.senthilal kumaran. et al [7] presented a hybrid method for white matter separation from MRI brain image that consist of three phase. First phase is to preprocess an image for segmentation, second phase is to segment an image using granular rough set and third phase is to separate white matter from segmented image using fuzzy sets This method was compared with mean shift algorithm and it was found that hybrid segmentation performs better result Rajesh patil et al [8] presented a method to detect and extract the tumor from patients MRI image of brain by using MATLAB software. This method performs noise removal function, Segmentation and morphological operations which are the basic concept of image processing. Tumour is extracted from MRI image for this it has an intensity more than that of its background so it becomes very easy locates. Mehdi Jafri and Reza Shafaghi [9] proposed a hybrid approach for detection of brain tumor tissue in MRI based on Genetic algorithm (GA) and support vector machine (SVM).In the preprocessing stage noise is removed and contrast is enhanced. For removing high frequency noises low pass filter is used for enhancing histogram stretching method is used. in segmentation undesired tissue such as nose, eyes and skull are deleted and features are extracted by different ways like FFT,GLCM and DWT. In feature selection GA is used with PCA by using this calculations complexity is reduced. Finally the selected features are applied to SVM classifier used to classify the image into normal or abnormal. A Sivaramkrishnan [10] presented a novel based approach in which Fuzzy C-mean (FCM) clustering algorithm was used to find the centroids of cluster groups to obtained brain tumor patterns. It is also preferred as faster clustering by using this centroid point can be located easily. The histogram equalization calculates the intensity of gray level image and PCA was used to reduce dimensionality of wavelet coefficient.

In the literature, there are a large number of existing techniques and algorithms for the detection and 
segmentation of brain tumor from MRI images. Asra Aslam et al. presented an improved edge detection algorithm for brain tumor segmentation [3]. This algorithm combines the Sobel method with image dependent thresholding, finds closed regions using closed contour algorithm and extracts tumors from the image. The brain tumors extracted by proposed algorithm are better than the tumors extracted using Sobel edge detector, Roberts edge detector and Prewitt edge detector. Roy et al. proposed a completely automatic algorithm to identify tumors by utilizing symmetry analysis [4]. In this paper, the idea that the region of image containing the tumor has higher intensity than the region with healthy brain tissues is being used. The MRI image is enhanced, filtered and segmentation is done and it also produces neighbor pixels for image segmentation [9]. The proposed algorithm uses the local statistical data to separate dependable neighbor pixels thereby improving the segmentation performance and the result of segmentation is adaptive to the original image. Gopinath et al. portrayed the proposed system for recognition and extraction of prostate cancer cells from the MRI image of the prostate organ [10]. In this paper, noise removal from MRI image by high pass and median filtering and then segmentation of MRI image is done by threshold segmentation, watershed segmentation and morphological operations. The extracted regions of cancerous cell from the input MRI image are extracted efficiently. R. B. Dubey et al. proposed a semi-automated region growing segmentation algorithm for the brain.

\section{PROPOSED METHOD}

\subsection{ROI BASED DICOM IMAGECOMPRESSION}

If we consider any medical image it requires large amount of data for storage and requires large transmission bandwidth, so we have to compress medical image.

A medical image for Compression can be a single image or sequence of images. The diagnostic data produced by hospitals has geometrically increased. Some of medical images which indicate that a compression technique is needed that results with greater data reductions and hence transmission speed.

In medical cases, a lossy compression method that preserves the diagnostic information is necessary. Recently ROI based coding has also been proved as a good approach for medical image compression especially in telemedicine applications.

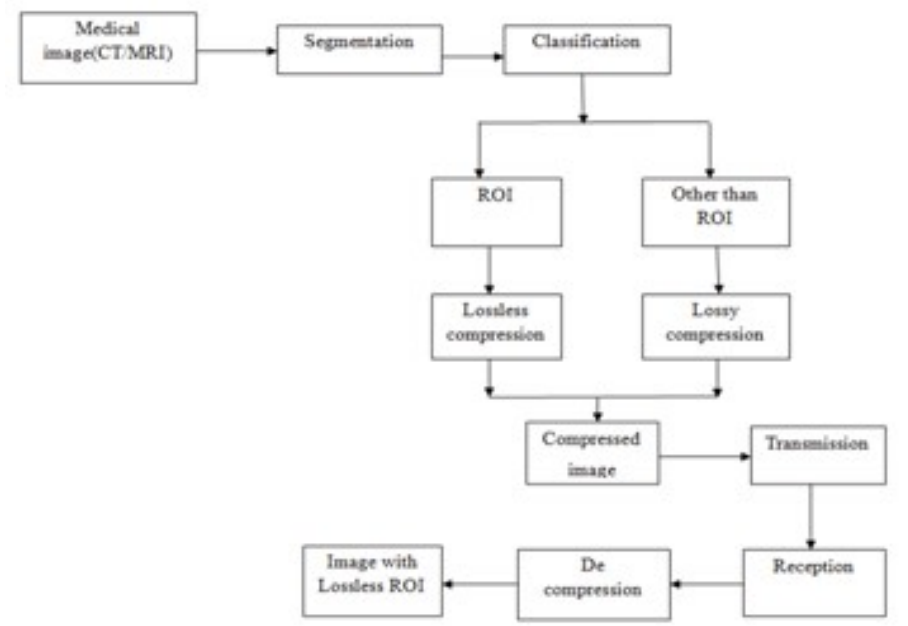

Fig.1 ROI based DICOM image compression

Region of interests (ROI) are those regions which can be given more importance in any given image.

If loss of quality is affordable, then many compression schemes produce high compression rates for general images. However, medicine cannot afford any deficiency in diagnostically important regions (ROI). Thus it is necessary to have an approach that brings a high compression rate maintaining good quality of the ROI. Since all regions of medical images do not have equal importance. Such as for brain MRI, instead of scanning the whole image the section of image that contains the tumor is examined. Which results in high reconstruction quality over user specified spatial regions in a limited time. Lossless compression, Progressive transmission and region of interest (ROI) are necessary requirements for a medical image compression scheme. In our proposed method, For the ROI based Image compression we have to separate both ROI and non ROI part and apply compression methods accordingly. In Fig 1 let us consider a medical image either $\mathrm{CT}$ or MRI and by applying segmentation technique the image will be divided into both ROI and non ROI parts.

\subsubsection{REGION OF INTEREST}

Those regions of an image which are given more consideration as compare to other regions are called region of interest i.e. ROI. It is a general observation that in some real image or medical image all the regions do not show equal importance for examination point of view. Considering this fact, attention is paid only to selected parts of the image. For example in Fig.1, of a brain MRI image, region containing tumor is examined instead of scanning the whole image, because actual information exists here. 
In medical diagnosis, the Region of Interest (ROI) concept is important because of the limitation and hampering of medical images due to lossy and lossless compression techniques. The compression ratio of lossless compression techniques result into $25 \%$ of original size, while for the lossy encoder $\mathrm{s}$ compression ratio is much greater, but both of these compressions causes loss in the data. This loss in data may cramp the important part of medical image. So to get rid from this problem, a better compression technique is needed which provide a better compression ratio by taking care of the important part (ROI) of the medical images.

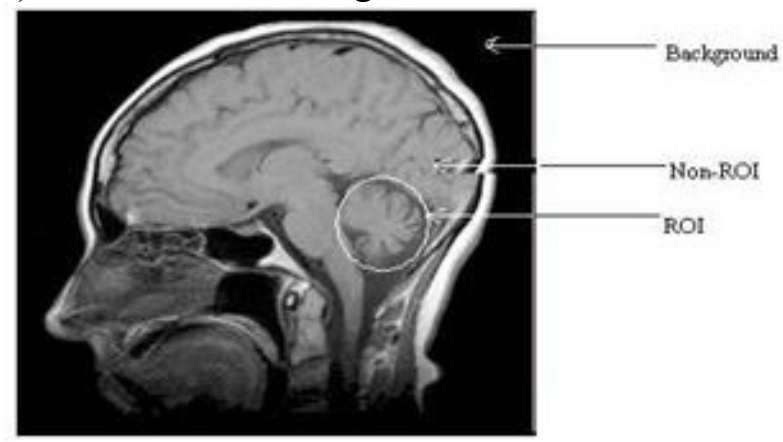

Fig 2 Different parts of a medical image

We will apply the lossless compression for ROI region by using Integer Wavelet Transform and apply lossy compression to non ROI part by using SPIHT algorithm. After that we will combined both the images that will be the compressed image. The compressed image will be transmitted through the transmission channel. At the receiver the image will be received and the original image will be extracted by doing decompression. The output image is the image with lossless ROI.

\subsubsection{SET PARTITIONING IN HIERARCHICAL TREES (SPIHT)}

In a wavelet-based still image coding algorithm known as set partitioning in hierarchical trees (SPIHT) is developed that generates a continuously scalable bit stream. This means that a single encoded bit stream can be used to produce images at various bit-rates and quality, without any drop in compression. The decoder simply stops decoding when a target rate or reconstruction quality has been reached. In the SPIHT algorithm, the image is first decomposed into a number of sub bands using hierarchical wavelet decomposition. The sub bands obtained for two-level decomposition are shown in Fig 2. The sub band coefficients are then grouped into sets known as spatial-orientation trees, which efficiently exploit the correlation between the frequency bands. The coefficients in each spatial orientation tree are then progressively coded bit-plane by biplane, starting with the coefficients with highest magnitude and at the lowest pyramid levels. Arithmetic coding can also be used to give further compression. In general, increasing the number of levels gives better compression although the improvement becomes negligible beyond 5 levels. In practice the number of possible levels can be limited by the image dimensions since the wavelet decomposition can only be applied to images with even dimensions. The use of arithmetic coding only results in a slight improvement for a 5 level decomposition.

The embedded zero tree wavelet (EZW) coding was first introduced by J.M. Shapiro and has since become a much studied topic in image coding. The EZW coding technique is a fairly simple and efficient technique for compressing the information in an image. Our focus in this project is to analyze the Set Partition in Hierarchical Tree algorithm in the EZW technique and to obtain observations by implementing the structure and testing it.

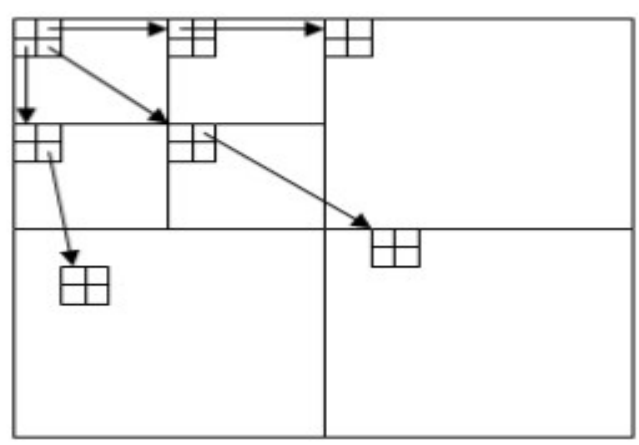

Fig 3 Two-level wavelet decomposition with orientation tree

In order to compress a binary file, some prior information must be known about the properties and structure of the file in order to exploit the abnormalities and assume the consistencies. The information that we know about the image file that is produced from wavelet transformation is that it can be represented in a binary tree format with the root of the tree having a much larger probably of containing a greater pixel magnitude level than that of the branches of the root. The algorithm that takes advantage of this information is the Set Partition in Hierarchical Tree (SPIHT) algorithm. 


\subsection{APPROACH}

Matlab offers a set of wavelet tools to be able to produce an image with the needed properties. The concept of wavelet transformation was not our focus in this project but in order to understand how the SPHT algorithm works; the properties of wavelet transformation would need to be identified. Matlab was able to create adequate testing pictures for this project. To adequately comprehend the advantages of the SPHT algorithm, a top level understanding will be needed to identify its characteristics and differences from other algorithms.

\subsection{SET PARTITIONING ALGORITHM}

The SPIHT algorithm is unique in that it does not directly transmit the contents of the sets, the pixel values, or the pixel coordinates. What it does transmit is the decisions made in each step of the progression of the trees that define the structure of the image. Because only decisions are being transmitted, the pixel value is defined by what points the decisions are made and their outcomes, while the coordinates of the pixels are defined by which tree and what part of that tree the decision is being made on. The advantage to this is that the decoder can have an identical algorithm to be able to identify with each of the decisions and create identical sets along with the encoder. The part of the SPIHT that designates the pixel values is the comparison of each pixel value to $2^{n} \leq\left|c_{\bar{i}, j}\right|<2^{n+1}$ with each pass of the algorithm having a decreasing value ofn.

In this way, the decoding algorithm will not need to passed the pixel values of the sets but can get that bit value from a single value of $n$ per bit depth level. This is also the way in which the magnitude of the compression can be controlled. By having an adequate number for $\mathrm{n}$, there will be many loops of information being passed but the error will be small, and likewise if $\mathrm{n}$ is small, the more variation in pixel value will be tolerated for a given signal pixel value. A pixel value that is $2^{n} \leq\left|c_{i, j}\right|$ is said to be significant for that pass. By sorting through the pixel values, certain coordinates can be tagged at "significant" or "insignificant" and then set into partitions of sets. The trouble with traversing through all pixel values multiple times to decide on the contents of each set is an idea that is inefficient and would take a large amount of time.

Therefore the SPIHT algorithm is able to make judgments by simulating a tree sort and by being able to only traverse into the tree as much as needed on each pass. This works exceptionally well because the wavelet transform produces an image with properties that this algorithm can take advantage of. This "tree" can be defined as having the root at the very upper left most pixel values and extending down into the image with each node having four ( $2 \times 2$ pixel group) of spring nodes. The SPIHT method is not an extension from the traditional methods of image compression, and it represents an important advance in the field. The SPIHT (set partitioning in hierarchical trees) is an efficient image coding method using the wavelet transform. Recently, image-coding using the wavelet transform has attracted great attention.

Among the many coding algorithms, the embedded zero tree wavelet coding by Shapiro and its improved version, the set partitioning in hierarchical trees (SPIHT) by Said and Pearlman have been very successful. Compared with JPEG which is the current standard for still image compression, the EZW and the SPIHT methods are more efficient and are able to reduce the blocking artifact.

The method provides the following which requires special attention

1. Good image quality and high PSNR especially for the color images

2. It is optimized for progressive image transmission

3. Produces a fully embedded coded file

4. Simple quantization algorithm

5. Can be used for lossless compression

6. Can code to exact bit rate or distortion

7. Fast coding/decoding (nearly symmetric)

8. Has wide applications, completely adaptive

Generally, different compression methods were developed that has at least one of the following properties but SPIHT really is outstanding since it has all those qualities simultaneously.

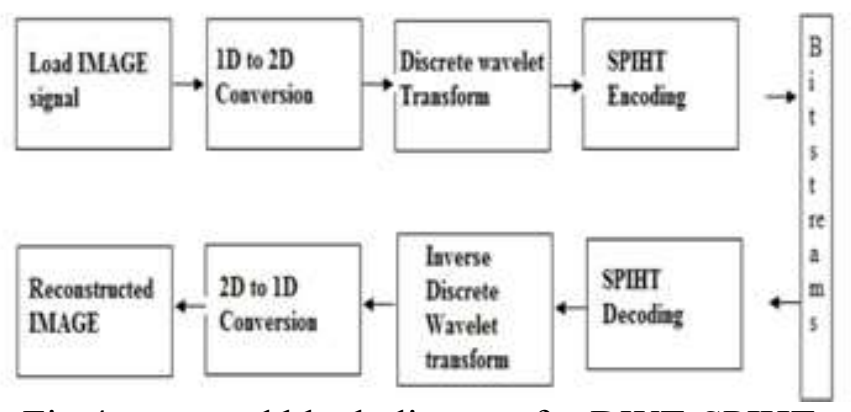

Fig 4 proposed block diagram for DWT-SPIHT encoding and decoding 
To make it simple, the following sets of coordinates are defined.

1. (i, j): set of coordinates of all offspring of node $(\mathrm{i}, \mathrm{j})$;

2. $\mathrm{D}(\mathrm{i}, \mathrm{j})$ : set of coordinates of all descendants of the node $(\mathrm{i}, \mathrm{j})$;

3. H: set of coordinates of all spatial orientation tree roots (nodes in the highest Pyramid level);

4. $L(i, j)=D(i, j)-O(i, j)$.

Thus, except at the highest and lowest levels, we have $\mathrm{O}(\mathrm{i}, \mathrm{j})=(2 \mathrm{i}, 2 \mathrm{j}),(2 \mathrm{i}, 2 \mathrm{j}+1),(2 \mathrm{i}+1,2 \mathrm{j}),(2 \mathrm{i}+1,2 \mathrm{j}+1)$

Define the following function

$$
s_{n}(\boldsymbol{\tau})=\left\{\begin{array}{l}
1, \max _{(i, j) \in \mathrm{r}}\left\{\left|c_{i, j}\right|\right\} \geq 2^{n} \\
0, \text { otherwise }
\end{array}\right.
$$

$s_{n}(\tau)$ Denotes the significance of a set of coordinates, where the preset significant Threshold used in the nth stage is denoted by $\mathrm{T}$ (n). The SPIHT coding algorithm is described as follows.

First, $\mathrm{T}(0)$ is assumed to $2^{M-1}$. Here $\mathrm{M}$ is chosen such that the largest coefficient magnitude $c_{\max }$, satisfies $\quad 2^{M-1} \leq c_{\max }<2^{n}$ in the coefficient magnitude, the encoding is progressive to successfully use a sequence of thresholds $2^{(M-1)-1}, n=0,1,2, \ldots M-1$. These thresholds are of the power of ' 2 ', so that the encoding can be taken as the bit plane encoding of the wavelet coefficients. All the coefficients with the magnitudes between $T(n)$ and $2 \mathrm{~T}(\mathrm{n})$, at stage $\mathrm{n}$ are significant and their positions and the sign bits are encoded. This is called sorting passprocess.

Then each coefficient with the magnitude at least $2 T(n)$ is refined by encoding the ' $n$ 'th most significant bit. This is known as refinement pass. The significant coefficient encoding position and the significant coefficient scanning can be achieved by the three lists: (LSP) the list of significant pixels, (LIP) the listof insignificant pixels, and (LIS) the listof insignificant set. Any entry into the LSP and LIP is an individual pixel which is represented or indicated by the coordinates $(i, j)$. Each entry into the LIS is regarded as the set: eitherD(i,j) or $\mathrm{L}(\mathrm{i}, \mathrm{j})$, such that LIS is indicated as type $A$ if it is $D(i, j)$ and type $B$ if it is $L(i, j)$.

\section{RESULTS}

In this paper we are showing of brain tumour extraction from the MRI scan images using matlab.

\subsection{INPUT MRI IMAGE FOR DETECTING TUMOUR}

The input image is MRI scanned image and which is in JPEG format ,here the tumour is detecting using matlab software.

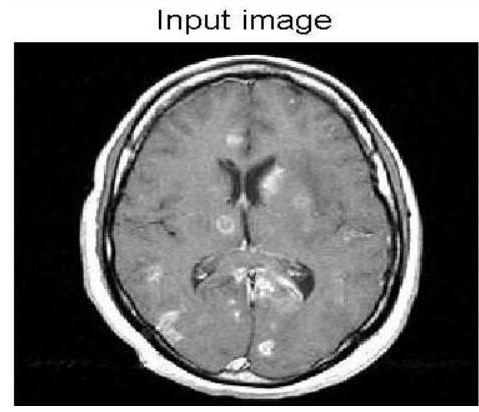

Fig 5.1 Input image

\subsection{CONVERTING INPUT IMAGE INTO GRAY SCALE IMAGE}

Generally gray scale images are preferred in the image-processing. Therefore the given input image was converting into the gray scale image.

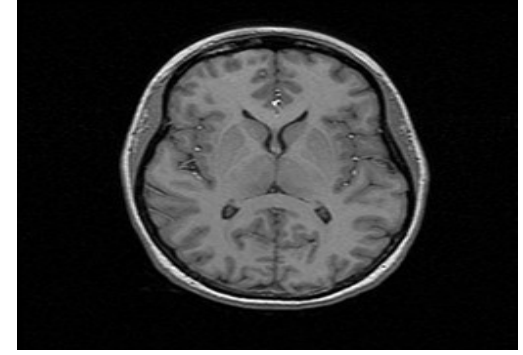

Fig 5.2 Gray scale image

\subsection{APPLYING HIGH PASS FILTER}

The gray scale image was given as input to the High pass filter for the sharpening the image .For the increasing the contrast of the image.

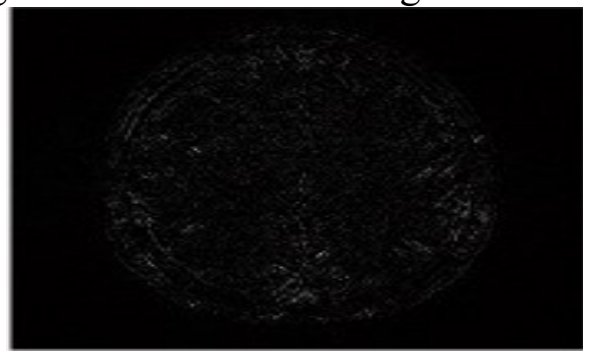

Fig 5.3 High pass filter image

\subsection{MEDAIN FILTER IMAGE}

The image from high pass filter was given to median filter for noise removal in the image.The output image from the median filter is below 


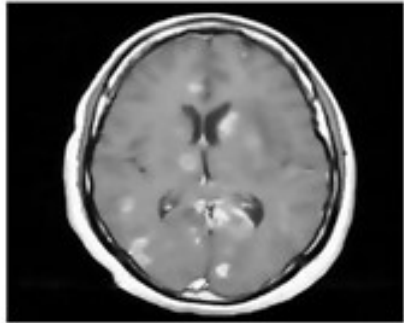

Fig 5.4 Median filter image

\subsection{TUMOUR DETECTING USING BOUNDING BOX}

The image from the median filter is given as input to the bounding box technology in the image Tumour in the brain image wsas decting using bounding box technology. The output image was below

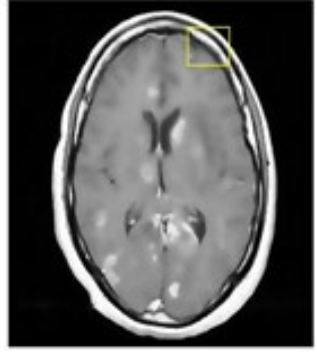

Fig 5.5 Bounding box detecting

\subsection{THRESHOLD SEGMENTATION OF IMAGE}

The output image from bounding box under goes to threshold segmentation the image was

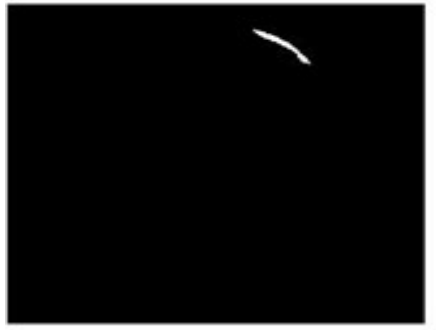

Fig 5.6 Threshold segmentation image

\subsection{WATERSHED SEGMENTATION IMAGE}

After thresholding the image undergoes watershed segmentation. the output image was below I umor vutıne

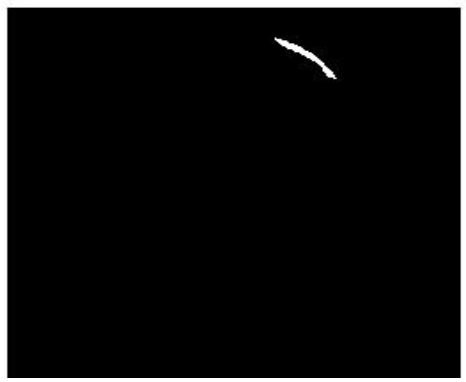

Fig 5.7 Watershed segmentation image

\subsection{OUTPUT OF TUMOUR DETECTION USING MATLAB}

In this process tumour detects by bounding box technology, the output is executed by using matlab code. The tumour was detected using gray scale convesion,high pass filter,median filter,threshold segmentation,watershed segmentation,morphological operation

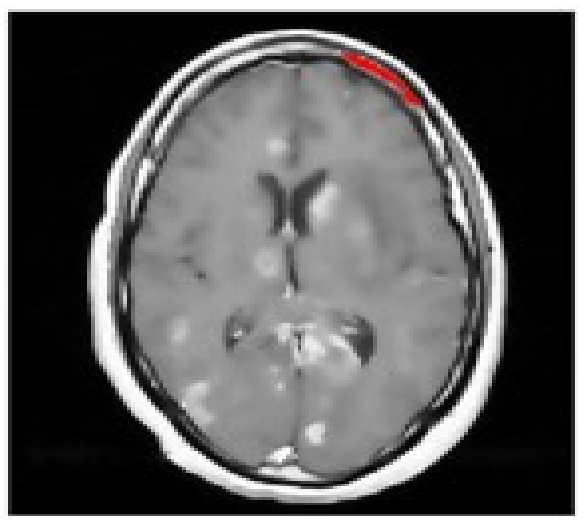

Fig 5.8 Tumor detected image

\section{CONCLUSION AND FUTURE SCOPE}

Every image contains some redundant information, which needs to be identified by the user to obtain compression. The floating point representation of the DWT gives small error in the system. The IWT is recommended for critical medical application because of its perfect reconstruction property. ROI-based compression is providing better results as compared with lossless methods, along with preservation of diagnostically important information. we have concluded that ROI based image compression is the best one. By this analysis we make sure that the compressed image will be helpful in telemedicine. After this compression we can send the medical image through mobile.

In near future, a database can be created for different patients having different types of brain tumours and locate them. Tumour growth can be analysed by plotting graph which can be obtained by studying sequential images of tumour affect. Possible extension of the presented work could use more features. It would be beneficial to connect the system to cloud storage of patient's information in hospital. This application can be extended to accessibility and usability through mobile phones. If this application is developed to analyze all types of MRI scans of same patient and result of all scans are integrated, it can suggest appropriate treatment and medication. 


\section{REFERENCES}

1. W. Gonzalez, "Digital Image Processing", 2nd ed. Prentice Hall, Year of Publication 2008

2. S. Murugavalli, V. Rajamani, "A high speed parallel fuzzy c-meanalgorithm for brain tumour segmentation"," BIME Journal”, Dec., 2006

3. Mohamed Lamine Toure, "Advanced Algorithm for Brain Segmentationusing Fuzzy to Localize Cancer and Epilepsy Region", InternationalConference on Electronics and Information Engineering (ICEIE 2010)

4. Dr.G.Padmavathi, Mr.M.Muthukumar and Mr. Suresh Kumar Thakur,"Non linear Image segmentation using fuzzy $\mathrm{c}$ means clustering methodwith thresholding for underwater images", IJCSI International Journal ofComputer Science Issues, May 2010

5. Matei Mancas, Bernard Gosselin, Benoît macq, "Segmentation Using aRegion Growing Thresholding"

6. T .Logeswari and M.Karnan "An improved implementation of braintumor detection using segmentation based on soft computing" Journal ofCancer Research and Experimental Oncology, March, 2010

7. Bairagi V K and Sapkal A M 2009 Selection of Wavelets for Medical ImageCompression.IEEE IntConf ACT 2009, Trivendrum, India, 678-680

8. Ali T J and Akhtar P 2008 Significance of region of interest applied on MRI and CT images in eleradiologytele medicine. Heidelberg: SpringerVerlag Berlin,151-159

9. Baeza I and Verdoy A 2009 ROI-based Procedures for progressive transmission of digital images: Acomparison. Elsevier J. Math. Comput.Model. 50:849-859

10. Bhaskaranarayana A, Satyamurthy L S, Murthy L N R, Sethuraman K and Rayappa H 2009 Bridging health divide between rural and urban areas - Satellite based telemedicine networks in India. Space Technologiesf or the Benefit of Human Society and Earth Part II,P. Olla (ed), Netherlands: SpringerBook Series, 160-178.

11. Dr. Krishnanaik Vankdoth "Research methods and presentation" Lap labmbert publishing, Germany in 2016.

\section{ABOUT THE AUTHORS}

Shri BOLISHETTI SATISH CHANDRA, Ph.D Research scholar from India and working as Associate
Professor in the Department of Electronics and communication Engineering, Mallareddy Institute of Engineering and Technology, Hyderabad. He studied B.Tech in Electronics and Communications engineering from Nagarjuna University, Guntur, AP and M.Tech in Systems and Signal Processing from JNTU college of engineering, JNTUH, Hyderabad, Telangana State. He is having $17+$ years of work experience in Academics, teaching and research. He participated and presented research papers in both national and international conference, seminars and work shops also published 7 research papers in national and international peer review journals.

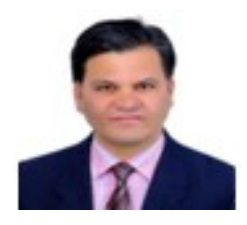

Dr. KRISHNANAIK VANKDOTH, currently working as a Professor, in the Department of Electronics \& Communication

Engineering,

Brilliant Grammar School Educational Society's group of institutions - Integrated Campus, Hyderabad. He studied B.E (ECE) from C. B. I. T, Osmania University, Hyderabad and M.Tech (Systems \& Signal Processing) from J.N.U.C, J.N.T.U, Hyderabad, Telangana, Ph.D (ECE) from Chittorgarh India. He is having $17+$ years of work experience in Academics, Teaching, and Industry \& Research. He participated and presented research papers in both national and international conferences, seminars and workshops; also published 24 Research papers in national and international peer reviewed journals, 4 Awards, 6 Academic Books international publications.

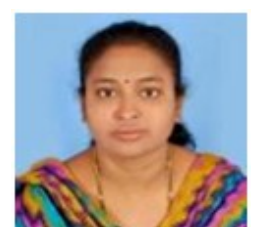

Smt K. SATYAVATHI Ph.D research scholar from India and working as Associate professor ,in the Department of Electronics and communication, Mallareddy institute of Engineering and technology, Hyd. She studied B.Tech in Electronics and Communications engineering from jntu University, hyderabad, AP and M.Tech in digital systems and computer electronics from JNTU college of engineering, JNTUH, Hyd,Telangana State .She is having $10+$ years of work experience in Academics ,teaching and research. She participated and presented research papers in both national and international conference, seminars and work shopsalso published 10 research papers in national and international peer review journals. 